\title{
Psychosocial Constraints on the Development of Resilience
}

\author{
ARNOLD J. SAMEROFF AND KATHERINE L. ROSENBLUM \\ Center for Human Growth and Development, University of Michigan, \\ Ann Arbor, Michigan, USA
}

\begin{abstract}
Although resilience is usually thought to reside in individuals, developmental research is increasingly demonstrating that characteristics of the social context may be better predictors of resilience. When the relative contribution of early resilience and environmental challenges to later child mental health and academic achievement were compared in a longitudinal study from birth to adolescence, indicators of child resilience, such as the behavioral and emotional self-regulation characteristic of good mental health, and the cognitive self-regulation characteristic of high intelligence contributed to later competence. However, the effects of such individual resilience did not overcome the effects of high environmental challenge, such as poor parenting, antisocial peers, low-resource communities, and economic hardship. The effects of single environmental challenges become very large when accumulated into multiple risk scores even affecting the development of offspring in the next generation.
\end{abstract}

KEYWORDS: resilience; adaptation; risk; mental health; intelligence; longitudinal studies

\section{DEFINING RESILIENCE}

Resilience connotes positive adaptation by individuals despite severe adversity. However, there has been sharp criticism concerning the construct of resilience and the methods used by resilience researchers. ${ }^{1}$ One of the main criticisms concerns the absence of a unifying conceptual framework that encompasses its integration across disciplines and specialized areas. A scientific basis for intervention research necessitates precise terminology to build upon earlier classifications and to ensure its continued vitality. A consistent and systematic framework is essential to facilitate the work of researchers and practitioners who pursue work in this area, to integrate findings across diverse

Address for correspondence: Arnold J. Sameroff, Center for Human Growth and Development, University of Michigan, 300 N. Ingalls Ann Arbor, MI 48105. Voice: 734-764-2443; fax: 734-9369288.

e-mail: sameroff@umich.edu

Ann. N.Y. Acad. Sci. 1094: 116-124 (2006). (C) 2006 New York Academy of Sciences. doi: 10.1196/annals.1376.010 
fields, as well as provide guidance for the identification and implementation of age-appropriate, optimal targets for preventive interventions. ${ }^{2}$

Although resilience is usually conceptualized as adaptive response to extraordinary challenge, it is not clear how it differs from adaptive responses to ordinary challenge except in some surprising ways. Extraordinary challenges can be separated into general and personal categories. In the general category are stressors that affect everyone in a community, such as war or earthquakes. Personal stressors are those specific to a particular family or individual, such as death or divorce.

What then are ordinary challenges? Essentially it is the stuff of life, the ordinary developmental stressors that individuals experience as they grow up. These again can be divided into general stressors, such as the educational systems and personal ones, such as coping with siblings. When children go to school, each curriculum is constructed as a series of challenges where as soon as the student overcomes one, such as learning to add, they are confronted with another, such as subtraction. In the family children must adapt to the requirements of others and the changes in relationships, for example, as their increasing autonomy skills are restricted by parental concerns with safety.

Is there evidence for a relation between responses to ordinary challenges and responses to extraordinary ones? The field of developmental psychopathology provides many examples where this seems to be the case. For several disorders that were thought to have a unique course, for example, acute schizophrenia, postpartum depression, and posttraumatic stress disorder, there is evidence that the amount of individual competence during the premorbid condition was highly predictive of the postmorbid resilience..$^{3-5}$

\section{OPERATIONALIZATION OF RESILIENCE}

Although a prescriptive definition of resilience may not be forthcoming, many of its ingredients have been identified including resourcefulness, intelligence, and mental health. Resourcefulness is almost a synonym for resilience with its ingredients of the ability to solve problems, to bounce back, and to learn from mistakes. The definition of intelligence has been broadening to include such elements as effective adaptation to the environment, learning from experience, and overcoming obstacles by taking thought. ${ }^{6}$

Modern conceptions of mental health include everyday adaptations to varying situations, which is the opposite of mental illness, which is the lack of everyday adaptation. Mental illness is the opposite of resilience in that behavior does not change with the situation. There is poor regulation of affect, behavior, and cognition. Healthy individuals may become saddened by the death of a relative but then move on, whereas depressives are saddened in all situations. Healthy children are hyperactive on the playground, but hyperactive children 
cannot regulate their behavior when they return to the classroom. Healthy individuals can attend to many different things but can focus down when it is a requirement of the task, but children with attention problems cannot adapt to the special situation. All these problems in self-regulation are evidence of a lack of resilience.

\section{OPERATIONALIZATION OF CHALLENGE}

Because resilience is defined as response to challenge, our next concern is to identify those factors that challenge individuals. Over the past three decades, studies of resilience have focused on individual variation in response to risky conditions, such as stressful life events, ${ }^{7}$ exposure to community violence, ${ }^{8}$ maltreatment, ${ }^{9}$ poverty, ${ }^{10}$ divorce,${ }^{11}$ and maternal mental illness. ${ }^{12}$ These studies have brought sharper attention to the social factors that influence stress resistance in children and adolescents.

Challenges are the equivalents of risk factors, defined as variables that increase the incidence of nonoptimal development. A representative set of such risk factors can be found in our longitudinal studies of families from the time target children were born until they were 30 years of age. From the data available in our study we chose a set of 10 social-environmental variables. ${ }^{13} \mathrm{We}$ then tested whether poor preschool cognitive and social-emotional resilience, defined here as competence, was related to the risk factors associated with low socioeconomic circumstances. The 10 environmental risk variables were: (1) a history of maternal mental illness, (2) high maternal anxiety, (3) parental perspectives that reflected rigidity in the attitudes, beliefs, and values that mothers had in regard to their child's development, (4) few positive maternal interactions with the child observed during infancy, (5) head of household in unskilled occupations, (6) minimal maternal education, (7) disadvantaged minority status, (8) single parenthood, (9) stressful life events, and (10) large family size. Each of these risk factors has a large literature documenting their potential for deleterious developmental effects, but there are many others not included in our list. Each of our 10 variables turned out to be a risk factor for preschool competence.

Because risk factors tend to cluster in the same individuals, many investigators soon realized that focusing on a single risk factor or challenge does not address the reality of most children's lives. ${ }^{14}$ As a way of improving predictive power, Rutter ${ }^{15}$ argued that it was not any particular risk factor but the number of risk factors in a child's background that led to psychiatric disorder. When we used this strategy in our study and created a multiple risk score that was the total number of risks for each individual family, major differences were found on mental health and intelligence measures between those children with few risks and those with many. On the intelligence test children with no environmental risks scored more than 30 points higher than children with 8 or 9 risk factors. ${ }^{13}$ No preschoolers in the zero-risk group had IQs below 85, 26\% 
of those in the high-risk group did. On average, each risk factor reduced the child's IQ score by 4 points. Four-year-olds in the high-risk group (5 or more risk factors) were 12.3 times as likely to be rated as having clinical mental health symptoms.

\section{CONTINUITY OF ENVIRONMENTAL CHALLENGE}

We completed new assessments of the sample when the children were 13 and 18 years of age. ${ }^{16}$ Because of the potent effects of our multiple risk index at 4 years, we calculated new multiple environmental risk scores for each family based on their situation 9 and 14 years later. To our surprise there were very few families that showed major shifts in the number of risk factors across the intervening periods.

The typical statistic reported in longitudinal research is the correlation between early and later performance of the children. We too found such correlations. Intelligence at 4 years correlated 0.72 with intelligence at 13 years and 0.67 from 13 to 18 years. The usual interpretation of such numbers is that there is a continuity of competence or incompetence in the child. Such a conclusion cannot be challenged if the only assessments in the study are of the children. In our study we examined and were able to correlate environmental challenges across time as well as child ones. We found a high correlation of 0.77 for environmental risk scores between 4 and 13 years and 0.80 between 13 and 18 years. These were greater than the continuities for the child measures. Whatever the child's ability for showing resilience and achieving higher levels of competence, it was severely undermined by the continuing number of environmental challenges. Whatever the capabilities provided to the child by individual factors, the environment seemed to limit further opportunities for development. Those children who had been living in high-risk environments at 4 years of age were still living in them at 13 and 18 years of age.

The 13- and 18-year high-risk contexts were producing the same negative effects on competence as the earlier 4-year ones had done. FIGURE 1 shows the relation between the IQ of these children and risk levels at the three assessments. The curves overlap substantially with similar ranges of outcomes at each age. We found the same relationship between the number of risk factors and the child's intellectual competence at each age; those children from families with no risk factors scored more than 30 points higher on intelligence tests than those with the most risk factors and had significantly better mental health.

\section{CAN INDIVIDUAL RESILIENCE OVERCOME SOCIAL CHALLENGE?}

We have demonstrated that the accumulation of environmental challenge has negative effects on a child's competence through adolescence. But what 


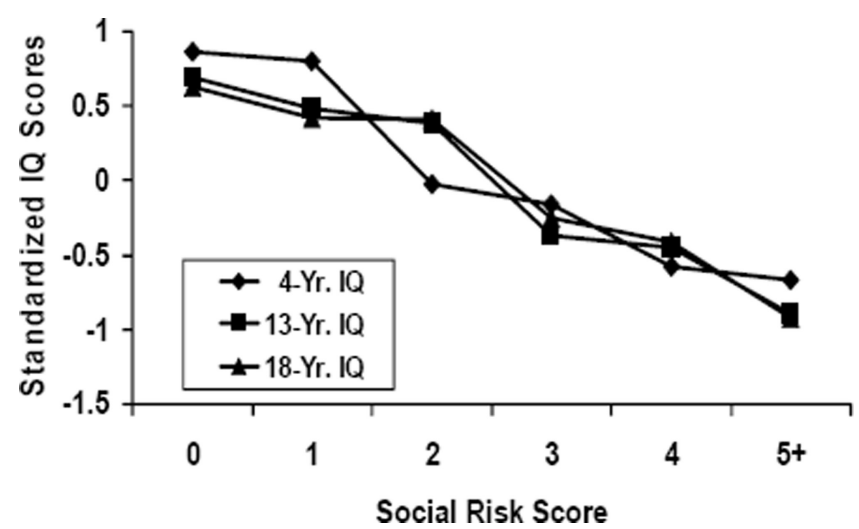

FIGURE 1. Relation of contemporary cumulative social risk score to standardized IQ scores at 4,13 , and 18 years of age.

about resilience? Would those children with higher levels of competence be better at overcoming social risk factors? Our study provided an opportunity to answer this question because we had measures of competence and challenge from infancy through adolescence. We could see how infant competence affected preschool competence, and then how preschool competence affected high school competence.

We started by testing whether infant competence could overcome challenges. For each infant we created a resilience or "multiple competence" score that included 12 factors from the data collected during the first year of life. Competence/resilience was defined as better scores on newborn medical and behavioral tests, temperament assessments, and developmental scales. We then divided the sample into groups of high and low resilient infants and examined as outcomes their 4-year IQ and social-emotional functioning scores to determine if these personal characteristics could overcome the negative effects of social adversity. We found no relation between infant competence and 4-year IQ or mental health problems. ${ }^{17}$ The single predictor of 4-year mental health and IQ was the multiple environmental risk score. Competent infants did no better than incompetent ones.

Infant developmental scales may be weak predictors because they assess different developmental functions than are captured by later cognitive and personality assessments. As many have argued, infant developmental scales do not incorporate aspects of mental functioning that are central to later IQ tests. Perhaps, if we move up the age scale we would find that the mental health and intelligence of these children at 4 years of age, when more cognitive functions are incorporated into IQ tests and greater social-emotional competence is possible, may be protective for later competence during adolescence. 
We divided the 4-year-olds into high and low mental health groups and high and low IQ groups in two analyses. We then compared these groups on how they did at 18 years on their mental health and measures of school achievement. Competent preschoolers did better on average than less competent ones but when we controlled for environmental risk, the differences between children with high and low levels of early competence paled when compared to the differences in performance between children in high- and low-risk social environments. We found that high competent children in high-risk environments did worse than low competent children in low-risk environments.

If 4-year competence is still too ephemeral to resist the negative consequences of adverse social circumstance, would competent 13-year-olds fare better at 18 years? We divided the 13-year-olds into high and low mental health groups and high and low intelligence groups, and examined their 18-year behavior. Again, in each case, 13-year-old youth with better mental health and intelligence did better within the same social risk conditions, but groups of children with high levels of competence living in conditions of high environmental risk did worse than low competent children in low-risk environments. ${ }^{18}$

\section{DEVELOPMENTAL TRAJECTORIES}

We have reported the effects of risk across two ages at a time, however, interactive processes between risk and protective factors often rely on chains of connections over time rather than on a multiplicative effect at any single time point. Understanding the factors that influence children's academic trajectories over many time points may help explain why some high-risk youth either catch up or fall further behind their more advantaged peers as they progress through school.

In our study we examined school records and obtained grades and attendance records for the participants from first to twelfth grade. We used hierarchical linear modeling (HLM) to examine the trajectories of the children throughout their school careers. ${ }^{19}$ We could then determine how the growth curves were influenced by their 4 -year multirisk scores. We could also determine the degree to which their early mental health and intelligence interacted with environmental risk to see if there was evidence of resilience in predicting school grades from first to twelfth grades.

These analyses confirmed our less sophisticated prior analyses: early risk had an adverse effect on academic trajectories during the entire period children were in school from the first to twelfth grades. For children in higher-risk families, having a higher IQ score was not a protective factor. There were no differences between the growth curves (see FIG. 2). But for children in lowrisk families, higher IQ was a promotive factor and resulted in a higher grade point trajectory. But consistent with earlier analyses, low resilient 4-year-olds 


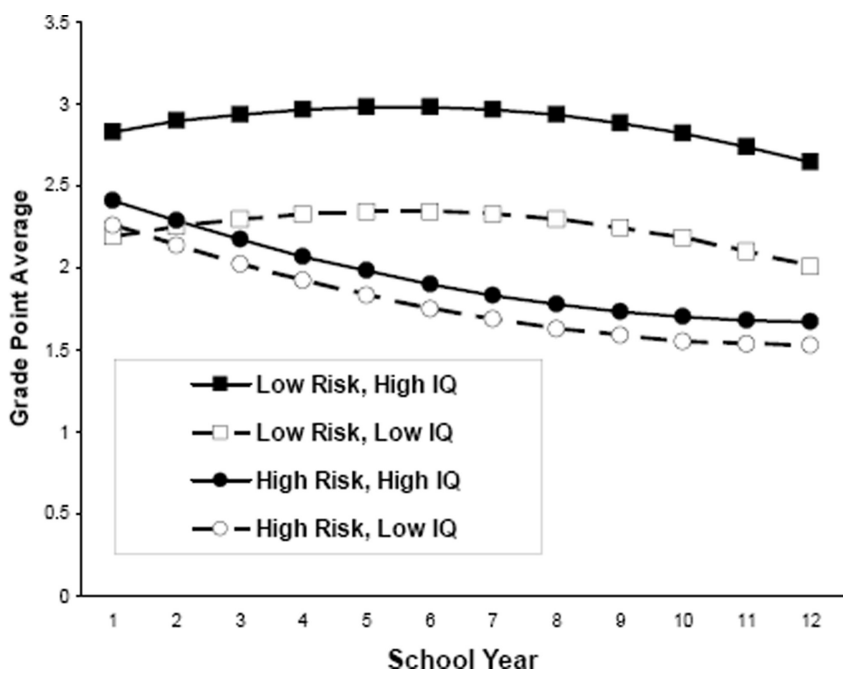

FIGURE 2. Effects of 4-year high and low cumulative social risk score and high and low 4-year verbal intelligence on trajectory of school grades (GPA) from first to twelfth grades (high is one standard deviation above the mean and low is one standard deviation below the mean).

in low-risk conditions consistently had higher GPAs than the high resilient children in high-risk conditions.

When we extend these analyses in time we find continuing predictive power of early risk factors on individual competence. The individuals in our study were contacted when they were 30 -year-olds and their mental health assessed using the DSM-IV-R global assessment of functioning (GAF) scores. Using regressions analyses we found that we could explain fully $30 \%$ of the variance

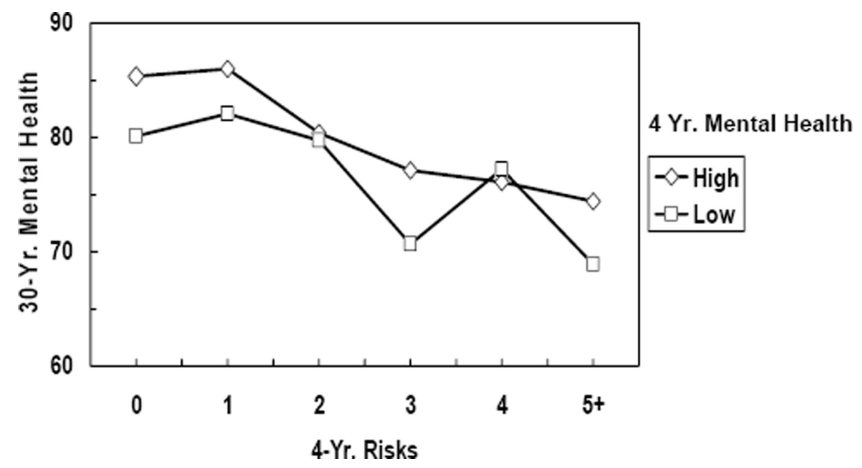

FIGURE 3. Relation of cumulative social risk score at 4 years of age to GAF at 30 years of age for 4-year high and low mental health groups. 
in their GAF scores over a span of 26 years. More to our point, the 4-year mental health measure of resilience only added $5 \%$ to the variance explained by the 4-year social risk score. FIGURE 3 shows the 30 -year GAF mental health scores for groups of high and low 4-year mental health groups as a function of number of 4-year risk scores. Here is another example where high-mental health 4-year-old children in high-risk conditions have worse 30-year mental health than low-mental health 4-year-old children in low-risk conditions.

\section{SUMMARY OF FINDINGS}

A focus on resilience as an individual characteristic does not explain more than a small proportion of variance in behavioral development. To truly appreciate the effects of childhood resilience requires attention to the broad constellation of social risk factors that challenge individuals and their families. ${ }^{20}$ The concern with preventing developmental failures has often clouded the fact that the majority of children in every social class and ethnic group are not failures. They get jobs, have successful social relationships, and raise a new generation of children. Masten ${ }^{20}$ concludes that the most surprising result of risk studies is the ordinariness of resilience and that it is both common and arises from the normative functions of human adaptational systems. In our analyses we have found that individual characteristics of mental health and higher intelligence contribute to developmental competence. However, the effects of such individual resiliencies do not overcome the effects of high environmental risk. In our analyses we consistently found that groups of high resilient children in highrisk environments had lower later mental health and cognitive competence than groups of low resilient children in low-risk environments.

\section{REFERENCES}

1. Luthar, S.S., D. CicChetTI, \& B. BeCKer. 2000. The construct of resilience: a critical evaluation and guidelines for future work. Child Dev. 71: 543-562.

2. SAMEROFF, A. \& L.M. GUTMAN. 2003. Contributions of risk research to the design of successful interventions. In Intervention with Children and Adolescents: An Interdisciplinary Perspective. P. Allen-Meares \& M.W. Fraser, Eds. New York: Pearson, Allyn \& Bacon.

3. Garmezy, N. \& E. Rodnick1959. Premorbid adjustment and performance in schizophrenia: implications for interpreting heterogeneity in schizophrenia. J. Nerv. Ment. Dis. 129: 450-466.

4. O'Hara, M.W. \& A.M. Swain. 1996. Rates and risk of postpartum depression-A meta-analysis. Int. Rev. Psychiatry 8: 37-54.

5. Breslau, N., E.L. Peterson, L.M. Poisson, et al. Estimating post-traumatic stress disorder in the community: lifetime perspective and the impact of typical traumatic events. Psychol. Med. 34: 889. 
6. Neisser, U., G. Boodoo, T.J. Bouchard, et al. 1996. Intelligence: knowns and unknowns. Am. Psychol. 51: 77-101.

7. Garmezy, N., A.S. Masten \& A. Tellegan. 1984. The study of stress and competence in children: a building block of developmental psychopathology. Child Dev. 55: 97-111.

8. White, K., S. Bruce, A. Farrell \& W. Kliewer. 1988. Impact of exposure to community violence on anxiety: a longitudinal study of family social support as a protective factor for urban children. J. Child Family Stud. 7: 187-203.

9. Cicchetti, D., S.L. Toth \& A. Maughan. 2000. An ecological-transactional model of child maltreatment. In Developmental Psychopathology. Vol 2. D. Cicchetti \& D.J. Cohen, Eds.: 689-722. Wiley. New York.

10. MCLOYD, V.C. 1997. Children in poverty: development, public policy, and practice. In Child Psychology in Practice. W. Damon, I.E. Siegel \& K.A. Renninger, Eds.: 135-208 Handbook of child psychology Fifth edition. Wiley. New York.

11. Hetherington, E.M. 2005. The influence of conflict, marital problem solving, and parenting on children's adjustment in nondivorced, divorced, and remarried families. In J. Families Count: Effects on Child and Adolescent Development. A. Clarke-Stewart \& J. Dunn. Ed.: Cambridge University Press. Cambridge, UK.

12. Sameroff, A.J., R. SeIfer \& M. ZaX. 1982. Early development of children at risk for emotional disorder. Monogr. Soc. Res. Child Dev. 47(7): 1-82.

13. Sameroff, A.J., R. Seifer, B. Barocas, M. Zax \& S. Greenspan. 1987. IQ scores of 4-year-old children: social-environmental risk factors. Pediatrics 79: 343350 .

14. Masten, A.S. \& J.D. Coatsworth. 1998. The development of competence in favorable and unfavorable environments: lessons from research on successful children. Am. Psychol. 53: 205-220.

15. RutTer, M. 1979. Protective factors in children's responses to stress and disadvantage. In Primary Prevention of Psychopathology (Vol. 3): Social Competence in Children. M.W. Kent \& J.E. Rolf, Eds.: University Press of New England. Hanover, NH.

16. Sameroff, A.J., R. Seifer, A. Baldwin \& C. Baldwin. 1993. Stability of intelligence from preschool to adolescence: the influence of social and family risk factors. Child Dev. 64: 80-97.

17. Sameroff, A.J. \& B.H. FieSE. 2000. Transactional regulation: The developmental ecology of early intervention. In Handbook of early childhood intervention. J.P. Shonkoff \& S.J. Meisels, Eds.: 135-159. Cambridge University Press. New York.

18. Sameroff, A.J., W.T. Bartko, A. Baldwin, et al. 1998. Family and social influences on the development of child competence. In Families, risk, and competence. M. Lewis \& C. Feiring, Eds.: Lawrence Erlbaum Associates. Mahwah, NJ.

19. Gutman, L.M., A.J. Sameroff \& R. Cole. 2003. Academic growth curve trajectories from first to twelfth grades: effects of multiple social risk and preschool child factors. Dev. Psychol. 39: 777-790.

20. Masten, A.S. 2001. Ordinary magic: resilience processes in development. Am. Psychol. 56: 227-238. 